\title{
Global convergence of a regularized factorized quasi-Newton method for nonlinear least squares problems
}

\author{
WEIJUN ZHOU ${ }^{\dagger}$ and LI ZHANG ${ }^{\ddagger}$ \\ College of Mathematics and Computational Science, \\ Changsha University of Science and Technology, Changsha 410004, China \\ E-mails: †weijunzhou@126.com / \$mathlizhang@126.com
}

\begin{abstract}
In this paper, we propose a regularized factorized quasi-Newton method with a new Armijo-type line search and prove its global convergence for nonlinear least squares problems. This convergence result is extended to the regularized BFGS and DFP methods for solving strictly convex minimization problems. Some numerical results are presented to show efficiency of the proposed method.
\end{abstract}

Mathematical subject classification: 90C53, 65K05.

Key words: factorized quasi-Newton method, nonlinear least squares, global convergence.

\section{Introduction}

The objective of this paper is to present a globally convergent factorized quasiNewton method for the nonlinear least squares problem

$$
\min f(x)=\frac{1}{2} \sum_{i=1}^{m} r_{i}^{2}(x)=\frac{1}{2}\|r(x)\|^{2}, \quad x \in R^{n} .
$$

Here $r(x)=\left(r_{1}(x), \cdots, r_{m}(x)\right)^{T}$ is called the residual function which is nonlinear and $\|\cdot\|$ denotes the Euclidean norm. It is easy to show that the gradient and the Hessian of $f$ are given by

$$
\nabla f(x)=J(x)^{T} r(x), \quad \nabla^{2} f(x)=J(x)^{T} J(x)+G(x),
$$


where $J(x)$ is the Jacobian matrix of $r(x)$,

$$
G(x)=\sum_{i=1}^{m} r_{i}(x) \nabla^{2} r_{i}(x)
$$

and $\nabla^{2} r_{i}(x)$ is the Hessian matrix of $r_{i}(x)$.

The nonlinear least squares problem has many applications in applied areas such as data fitting and nonlinear regression in statistics. Many efficient algorithms have been proposed to solve this problem. The special structure of the Hessian leads to a lot of special methods which have different convergence properties for zero and nonzero residual problems $[5,6,8,20]$.

The Gauss-Newton method and the Levenberg-Marquardt method are two well-known methods which have locally quadratic convergence rate for zero residual problems [6]. However, they may converge slowly or even diverge when the problem is nonzero residual or the residual function is highly nonlinear [1]. The main reason is that both methods only use the first order information of $f$.

To solve nonzero residual problems more efficiently, some structured quasiNewton methods using the second order information of $f$ have been proposed. These methods are shown to be superlinearly convergent for both zero and nonzero residual problems $[5,8]$. However, the iterative matrices of structured quasi-Newton methods are not necessarily positive definite. Therefore the search directions may not be descent directions of $f$ when some line search is used.

To guarantee the positive definite property of the iterative matrices, some factorized quasi-Newton methods have been proposed [15, 16, 18, 19, 20, 21, 22], where the search direction is given by

$$
\left(J_{k}+L_{k}\right)^{T}\left(J_{k}+L_{k}\right) d=-J_{k}^{T} r_{k},
$$

where $J_{k}=J\left(x_{k}\right), r_{k}=r\left(x_{k}\right)$ and $L_{k}$ is updated according to certain quasiNewton formula. These methods have been proved to be superlinearly convergent for both zero and nonzero residual problems. But they may not possess quadratical convergence rate for zero residual problems. Based on the idea of [10], Zhang et al. [24] proposed a family of scaled factorized quasiNewton methods which not only have superlinear convergence rate for nonzero residual problems, but also have quadratical convergence rate for zero residual 
problems. Under suitable conditions, the iterative matrices of factorized quasiNewton methods are proved to be positive definite if the initial point is close to the solution point $[20,24]$.

However, all iterative matrices of factorized quasi-Newton methods may not be positive definite if the initial point is far from the solution. Therefore, the search direction may not be descent. This is a drawback of factorized quasiNewton methods to have global convergence when the line search methods are used. Another difficulty for the global convergence is that the iterative matrices and their inverses may not be uniformly bounded. To the best of our knowledge, global convergence of factorized quasi-Newton methods has not been established.

The paper is organized as follows. In Section 2, we propose a regularized factorized quasi-Newton method which guarantees that the iterative matrix is positive definite at each step. We use a new Armijo-type line search to compute the stepsize. Under suitable conditions, we prove that the proposed method converges globally for nonlinear least squares problems. In Section 3, we extend this result to the BFGS and DFP methods for general nonlinear optimization. We show that the regularized BFGS and DFP methods converge globally for strictly convex objective functions. In Section 4, we compare the performance of the proposed method to some existing methods and present some numerical results to show efficiency of the proposed method.

\section{Algorithm and global convergence}

In this section, we first present the regularized factorized quasi-Newton algorithm and then analyze its global convergence property. The motivation of the method is that the positive definite property of the iterative matrix can be guaranteed by the use of the Levenberg-Marquardt regularization technique. It is important for global convergence of the method to choose suitable regularization parameter and stepsize carefully. Now we give the details of the method.

Algorithm 2.1 (Regularized factorized quasi-Newton method)

Step 1. Give the starting point $x_{0} \in R^{n}, L_{0} \in R^{m \times n}, \delta, \rho \in(0,1)$, $\epsilon_{1}>0, \epsilon_{2}>0, r \geq 0, M>0$. Choose a positive constant $\beta>\frac{1}{1-\delta}>1$. 
Let $k:=0$.

Step 2. Compute $d_{k}$ by solving the linear equations

$$
\left(B_{k}+\mu_{k} I\right) d=-g_{k},
$$

where

$$
B_{k}=\left(J_{k}+L_{k}\right)^{T}\left(J_{k}+L_{k}\right), \quad g_{k}=J_{k}^{T} r_{k}
$$

and

$$
\mu_{k}= \begin{cases}\epsilon_{1}\left\|B_{k}\right\| & \text { if }\left\|B_{k}\right\|>\max \left(M, \frac{1}{\left\|g_{k}\right\|}\right), \\ \epsilon_{2}\left\|g_{k}\right\|^{r} & \text { otherwise. }\end{cases}
$$

Here $\left\|B_{k}\right\|$ is referred to the Frobenius norm of $B_{k}$. For simplicity, we denote

$$
\begin{aligned}
& K_{1} \triangleq\left\{k \mid\left\|B_{k}\right\|>\max \left(M, \frac{1}{\left\|g_{k}\right\|}\right)\right\}, \\
& K_{2} \triangleq\left\{k \mid\left\|B_{k}\right\| \leq \max \left(M, \frac{1}{\left\|g_{k}\right\|}\right)\right\} .
\end{aligned}
$$

Step 3. (i) If $k \in K_{2}$, compute stepsize $\alpha_{k}=\max \left\{\rho^{0}, \rho^{1}, \cdots\right\}$ such that

$$
f\left(x_{k}+\alpha_{k} d_{k}\right) \leq f\left(x_{k}\right)+\delta \alpha_{k} g_{k}^{T} d_{k} .
$$

(ii) If $k \in K_{1}$, we consider the following two cases.

Case (1): $f\left(x_{k}+d_{k}\right) \leq f\left(x_{k}\right)+\delta g_{k}^{T} d_{k}$. If $f\left(x_{k}+\beta d_{k}\right)>f\left(x_{k}+d_{k}\right)+\delta \beta g_{k}^{T} d_{k}$, set $\alpha_{k}=1$; Otherwise, compute stepsize $\alpha_{k}=\max \left\{\beta^{1}, \beta^{2}, \cdots\right\}$ satisfying

$$
f\left(x_{k}+\beta^{m} d_{k}\right) \leq f\left(x_{k}+\beta^{m-1} d_{k}\right)+\delta \beta^{m} g_{k}^{T} d_{k} .
$$

Case (2): $f\left(x_{k}+d_{k}\right)>f\left(x_{k}\right)+\delta g_{k}^{T} d_{k}$. Compute stepsize $\alpha_{k}=\max \left\{\rho^{1}, \cdots\right\}$ such that

$$
f\left(x_{k}+\rho^{m} d_{k}\right) \leq f\left(x_{k}\right)+\delta \rho^{m} g_{k}^{T} d_{k} .
$$

Step 4. Set $x_{k+1}=x_{k}+\alpha_{k} d_{k}$. Update $L_{k}$ to get $B_{k+1}=\left(J_{k+1}+\right.$ $\left.L_{k+1}\right)^{T}\left(J_{k+1}+L_{k+1}\right)$ by certain quasi-Newton formula. Let $k:=k+1$ and go to Step 2. 
Remark 2.1. (i) Since the matrix $B_{k}$ is positive semidefinite, the iterative matrix $B_{k}+\mu_{k} I$ is positive definite, which ensures that the search direction $d_{k}$ is a descent direction, that is, $g_{k}^{T} d_{k}<0$. The choice of $\mu_{k}$ is based on the ideas of $[9,12,23]$.

(ii) The line search (2.4) is different from the standard Armijo line search (2.3) since $\beta>1$ in (2.4). This new Armijo-type line search can accept stepsize as large as possible in Case (1). The following proposition shows that it is well-defined.

Proposition 2.1. The Algorithm 2.1 is well-defined.

Proof. We only need to prove that the line search (2.4) terminates finitely. If it is not true, then for infinite many $m$, we have

$$
\frac{f\left(x_{k}+\beta^{m} d_{k}\right)}{\beta^{m}} \leq \frac{f\left(x_{k}+\beta^{m-1} d_{k}\right)}{\beta^{m}}+\delta g_{k}^{T} d_{k} .
$$

Let $m \rightarrow \infty$ in the above inequality, then from $\beta>1, f(x) \geq 0$ for all $x$ and $f\left(x_{k}+\beta^{m} d_{k}\right) \leq f\left(x_{k}\right) \leq f\left(x_{0}\right)$, we have

$$
g_{k}^{T} d_{k}>0,
$$

which leads to a contradiction.

In the convergence analysis of Algorithm 2.1, we need the following assumption.

\section{Assumption A.}

(I) The level set $\Omega=\left\{x \in R^{n} \mid f(x) \leq f\left(x_{0}\right)\right\}$ is bounded.

(II) In some neighborhood $N$ of $\Omega, f$ is continuously differentiable and its gradient is Lipschitz continuous, namely, there exists a constant $L>0$ such that

$$
\|g(x)-g(y)\| \leq L\|x-y\|, \quad \forall x, y \in N
$$


It is clear that the sequence $\left\{x_{k}\right\}$ generated by Algorithm 2.1 is contained in $\Omega$. Moreover, the sequence $\left\{f\left(x_{k}\right)\right\}$ is a descent sequence. Therefore it has a limit $f^{*}$, that is,

$$
\lim _{k \rightarrow \infty} f\left(x_{k}\right)=f^{*} .
$$

In addition, from Assumption A we get that there is a positive constant $\gamma$ such that

$$
\|g(x)\| \leq \gamma, \quad \forall x \in \Omega .
$$

Now we give some useful lemmas for the convergence analysis of the algorithm.

Lemma 2.2. Let Assumption A hold. Then we have

$$
\lim _{k \rightarrow \infty} \alpha_{k} g_{k}^{T} d_{k}=0
$$

Proof. It follows directly from (2.3), (2.4), (2.5) and (2.7).

For convenience, we denote index sets

$$
K_{3} \triangleq\left\{k \mid \alpha_{k} \text { satisfies (2.4) }\right\}, \quad K_{4} \triangleq\left\{k \mid \alpha_{k} \text { satisfies (2.5) }\right\} .
$$

Then we have

$$
K_{1}=K_{3} \cup K_{4}
$$

Lemma 2.3. If $k \in K_{1}$, then there exists a constant $c_{1}>0$ such that

$$
\alpha_{k} \geq c_{1} \frac{-g_{k}^{T} d_{k}}{\left\|d_{k}\right\|^{2}}
$$

Proof. If $k \in K_{3}$, then from the line search (2.4), we have

$$
f\left(x_{k}+\alpha_{k} \beta d_{k}\right)>f\left(x_{k}+\alpha_{k} d_{k}\right)+\delta \alpha_{k} \beta g_{k}^{T} d_{k} .
$$

By the mean values theorem and (2.6), we have

$$
f\left(x_{k}+\alpha_{k} \beta d_{k}\right)-f\left(x_{k}+\alpha_{k} d_{k}\right) \leq \alpha_{k}(\beta-1) g_{k}^{T} d_{k}+L\left[\alpha_{k}(\beta-1)\right]^{2}\left\|d_{k}\right\|^{2} .
$$


The above two inequalities implies

$$
\alpha_{k} \geq c_{1} \frac{-g_{k}^{T} d_{k}}{\left\|d_{k}\right\|^{2}}
$$

where $c_{1}=\frac{\beta(1-\delta)-1}{L(\beta-1)^{2}}>0$. Here we use the conditions $\beta>\frac{1}{1-\delta}$ and $\delta \in(0,1)$.

If $k \in K_{4}$, then from the line search (2.5), we have

$$
f\left(x_{k}+\frac{\alpha_{k}}{\rho} d_{k}\right)>f\left(x_{k}\right)+\delta \frac{\alpha_{k}}{\rho} g_{k}^{T} d_{k} .
$$

Therefore the inequality (2.10) also holds by using similar proof in the case $k \in K_{3}$. The proof is then completed since $K_{1}=K_{3} \cup K_{4}$.

Lemma 2.4. If $k \in K_{2}$, then there exists a constant $c_{2}>0$ such that

$$
\alpha_{k}=1, \quad \text { or } \quad \alpha_{k} \geq c_{2} \frac{-g_{k}^{T} d_{k}}{\left\|d_{k}\right\|^{2}} .
$$

Proof. For $k \in K_{2}$, if $\alpha_{k} \neq 1$, then by the line search (2.3), we also have

$$
f\left(x_{k}+\frac{\alpha_{k}}{\rho} d_{k}\right)>f\left(x_{k}\right)+\delta \frac{\alpha_{k}}{\rho} g_{k}^{T} d_{k} .
$$

Then the conclusion follows directly from the same argument as Lemma 2.3.

The proof of the following lemma is similar to that of Theorem 2.2.2 of [12], for completeness, we present the proof here.

Lemma 2.5. Let Assumption A hold and the sequence $\left\{x_{k}\right\}$ be generated by Algorithm 2.1. If $K_{1}$ is infinite, then we have

$$
\liminf _{k \rightarrow \infty}\left\|g_{k}\right\|=0
$$

Proof. If $k \in K_{1}$, then $\mu_{k}=\epsilon_{1}\left\|B_{k}\right\|$. Now we set $\bar{\alpha}_{k}=\alpha_{k} /\left\|B_{k}\right\|$ and $\bar{d}_{k}=$ $\left\|B_{k}\right\| d_{k}$, then we have

$$
\bar{\alpha}_{k} \bar{d}_{k}=\alpha_{k} d_{k}
$$


We have from (2.1) that

$$
\begin{aligned}
-g_{k}^{T} \bar{d}_{k} & =d_{k}^{T} B_{k} \bar{d}_{k}+\epsilon_{1}\left\|B_{k}\right\| d_{k}^{T} \bar{d}_{k} \\
& =d_{k}^{T} B_{k} \bar{d}_{k}+\epsilon_{1}\left\|\bar{d}_{k}\right\|^{2} \geq \epsilon_{1}\left\|\bar{d}_{k}\right\|^{2} .
\end{aligned}
$$

From Lemma 2.3 and (2.12), we get

$$
\bar{\alpha}_{k}\left\|\bar{d}_{k}\right\|^{2}=\alpha_{k} d_{k}^{T} \bar{d}_{k} \geq c_{1} \frac{-g_{k}^{T} d_{k}}{\left\|d_{k}\right\|^{2}} d_{k}^{T} \bar{d}_{k}=c_{1}\left(-g_{k}^{T} \bar{d}_{k}\right) .
$$

This inequality together with (2.13) shows that

$$
\bar{\alpha}_{k} \geq c_{1} \epsilon_{1}
$$

It follows from (2.9) and (2.12) that

$$
\lim _{k \rightarrow \infty} \bar{\alpha}_{k} g_{k}^{T} \bar{d}_{k}=\lim _{k \rightarrow \infty} \alpha_{k} g_{k}^{T} d_{k}=0 .
$$

The inequalities (2.15), (2.16) and (2.13) imply that

$$
\lim _{k \rightarrow \infty, k \in K_{1}} \bar{d}_{k}=0 .
$$

Therefore from the above equality and (2.1) we have

$$
\begin{aligned}
\lim _{k \rightarrow \infty, k \in K_{1}}\left\|g_{k}\right\| & \leq \lim _{k \rightarrow \infty, k \in K_{1}}\left\|B_{k} d_{k}+\mu_{k} d_{k}\right\| \\
& \leq \lim _{k \rightarrow \infty, k \in K_{1}}\left(\left\|B_{k}\right\|\left\|d_{k}\right\|+\epsilon_{1}\left\|B_{k}\right\|\left\|d_{k}\right\|\right) \\
& =\lim _{k \rightarrow \infty, k \in K_{1}}\left(1+\epsilon_{1}\right)\left\|\bar{d}_{k}\right\|=0 .
\end{aligned}
$$

The proof is then finished.

The following theorem shows that Algorithm 2.1 is globally convergent.

Theorem 2.6. Let Assumption A hold and the sequence $\left\{x_{k}\right\}$ be generated by Algorithm 2.1, then we have

$$
\liminf _{k \rightarrow \infty}\left\|g_{k}\right\|=0 .
$$


Proof. We suppose that the conclusion of the theorem is not true. Then there exists a constant $\varepsilon>0$ such that for any $k \geq 0$, it holds that

$$
\left\|g_{k}\right\| \geq \varepsilon
$$

From Lemma 2.5, we only need to consider the case that $K_{1}$ is finite. Therefore there exists $k_{0}>0$ such that for all $k>k_{0}, \mu_{k}=\epsilon_{2}\left\|g_{k}\right\|^{r}$.

It follows from (2.1) and (2.17) that

$$
-g_{k}^{T} d_{k}=d_{k}^{T} B_{k} d_{k}+\epsilon_{2}\left\|g_{k}\right\|^{r}\left\|d_{k}\right\|^{2} \geq \epsilon_{2} \gamma^{r}\left\|d_{k}\right\|^{2} .
$$

This inequality together with Lemma 2.4 and (2.9) means that

$$
\lim _{k \rightarrow \infty}\left\|d_{k}\right\|=0 .
$$

From (2.1), (2.17) and the definition of $K_{2}$, we have

$$
\begin{aligned}
\mid g_{k}\|=\| B_{k} d_{k}+\mu_{k} d_{k} \| & \leq\left(\left\|B_{k}\right\|+\epsilon_{2}\left\|g_{k}\right\|^{r}\right)\left\|d_{k}\right\| \\
& \leq\left(\max \left(M, \frac{1}{\varepsilon}\right)+\epsilon_{2} \gamma^{r}\right)\left\|d_{k}\right\|
\end{aligned}
$$

It follows from the above inequality and (2.18) that

$$
\lim _{k \rightarrow \infty}\left\|g_{k}\right\|=0,
$$

which contradicts with (2.17). This finishes the proof.

\section{Application to nonlinear optimization}

In this section, we will extend the result of Section 2 to the BFGS method and the DFP method for the general nonlinear optimization problem

$$
\min f(x), \quad x \in R^{n},
$$

where $f: R^{n} \rightarrow R$ is continuously differentiable. 
The BFGS method and the DFP method are two well-known quasi-Newton methods for solving (3.1). Their updated formulas are given by

$$
\begin{array}{ll}
\text { BFGS formula: } & B_{k+1}=B_{k}-\frac{B_{k} s_{k} s_{k}^{T} B_{k}}{s_{k}^{T} B_{k} s_{k}}+\frac{y_{k} y_{k}^{T}}{y_{k}^{T} s_{k}} \\
\text { DFP formula: } & B_{k+1}=\left(I-\frac{y_{k} s_{k}^{T}}{y_{k}^{T} s_{k}}\right) B_{k}\left(I-\frac{s_{k} y_{k}^{T}}{y_{k}^{T} s_{k}}\right)+\frac{y_{k} y_{k}^{T}}{y_{k}^{T} s_{k}} ;
\end{array}
$$

where $y_{k}=g_{k+1}-g_{k}$, and $s_{k}=x_{k+1}-x_{k}$. An important property of both methods is that $B_{k+1}$ can inherit the positive definiteness of $B_{k}$ if $s_{k}^{T} y_{k}>0$ [6]. If $f$ is strictly convex or the Wolfe line search is used, then $s_{k}^{T} y_{k}>0$ and $B_{k+1}$ is well-defined.

During the past three decades, global convergence of the BFGS and DFP methods has received growing interests. When $f$ is convex and the exact line search is used, Powell proved that both methods converge globally [17]. When the Wolfe line search is used, Byrd et al. [3] proved the global convergence of the convex Broyden's class except for the DFP method. Byrd and Nocedal [2] obtained global convergence of the BFGS method with the standard Armijo line search for strongly convex function. For nonconvex optimization, counterexamples in $[4,13]$ show that the BFGS method may not converge globally when using exact line search or the Wolfe line search.

Li and Fukushima [11] proposed a modified BFGS method which possesses global and superlinear convergence even for nonconvex functions. Zhou and Zhang [25] extended this result to the nonmonotone case. Zhou and Li [26] proposed a modified BFGS method for nonlinear monotone equations and established its global convergence. But these modified BFGS formulas destroy the affine invariability of the standard BFGS formula. To overcome this drawback, Liu [12] proposed a regularized BFGS method and proved that this method converges globally for nonconvex functions if the Wolfe line search is used. Liu [12] also proposed a question that whether the regularized BFGS method is globally convergent for strictly convex or nonconvex functions if Armijo-type line search is used. The answer is positive.

In fact, if we update $B_{k}$ in Algorithm 2.1 by the BFGS update foumula or DFP update formula, then a direct consequence of Theorem 2.6 is that the regularized BFGS and DFP methods converge globally for strictly convex functions. 
Corollary 3.1. Let Assumption A hold. If $f$ is strictly convex, then for the regularized BFGS and DFP methods, we have $\lim _{\inf } \operatorname{lo}_{k \rightarrow \infty}\left\|g_{k}\right\|=0$.

\section{Numerical experiments}

In this section we compare the number of iterations and function evaluations performance of the proposed method to some existing methods such as the GausssNewton method for nonlinear least squares problems. The details of six methods are listed as follows.

(1) The Gauss-Newton method, denoted GN, which has the search direction given by $B_{k} d=-g_{k}, B_{k}=J_{k}^{T} J_{k}$. We compute the stepsize by the standard Armijo line search, that is, compute stepsize $\alpha_{k}=\max \left\{\rho^{0}, \rho^{1}, \cdots\right\}$ satisfying

$$
f\left(x_{k}+\rho^{m} d_{k}\right) \leq f\left(x_{k}\right)+\delta \rho^{m} g_{k}^{T} d_{k},
$$

where we choose $\delta=0.1, \rho=0.5$.

(2) The Levenberg-Marquardt method, denoted LM, whose search direction is defined by $B_{k} d=-g_{k}, B_{k}=J_{k}^{T} J_{k}+\mu_{k} I, \mu_{k}=\left\|g_{k}\right\|$. The stepsize is computed by (4.1).

(3) The factorized BFGS method [20], denoted FBFGS, whose search direction is given by $B_{k} d=-g_{k}$, and $B_{k}$ is updated by

$$
B_{k+1}=\left(J_{k+1}+L_{k+1}\right)^{T}\left(J_{k+1}+L_{k+1}\right)
$$

where

$$
\begin{aligned}
& L_{k+1}=L_{k}+\frac{\bar{L}_{k} s_{k}}{s_{k}^{T} \bar{B}_{k} s_{k}}\left(\left(\frac{s_{k}^{T} \bar{B}_{k} s_{k}}{s_{k}^{T} z_{k}}\right)^{1 / 2} z_{k}-\bar{B}_{k} s_{k}\right)^{T}, \\
& \bar{L}_{k}=J_{k+1}+L_{k}, \\
& \bar{B}_{k}=\bar{L}_{k}^{T} \bar{L}_{k}, \\
& z_{k}=\left(J_{k+1}-J_{k}\right)^{T} r\left(x_{k+1}\right)+J_{k+1}^{T} J_{k+1} s_{k} .
\end{aligned}
$$

The stepsize is obtained by (4.1). 
(4) Algorithm 2.1, denoted R-FBFGS, where $B_{k}$ is updated by (4.2). Here we use the following values for the parameters: $\delta=0.1, \rho=0.5, \beta=2$, $M=10^{4}, \epsilon_{1}=10^{-8}, \epsilon_{2}=1$ and $r=1$.

(5) The scaled factorized BFGS method [24], denoted SFBFGS, whose search direction is given by $B_{k} d=-g_{k}$, and $B_{k}$ is updated by

$$
B_{k+1}=\left(J_{k+1}+\left\|r\left(x_{k+1}\right)\right\| L_{k+1}\right)^{T}\left(J_{k+1}+\left\|r\left(x_{k+1}\right)\right\| L_{k+1}\right)
$$

where

$$
\begin{aligned}
& L_{k+1}=\frac{\left\|r\left(x_{k+1}\right)\right\|}{\left\|r\left(x_{k}\right)\right\|} L_{k}+\frac{1}{\left\|r\left(x_{k+1}\right)\right\|} \frac{\bar{L}_{k} s_{k}}{s_{k}^{T} \bar{B}_{k} s_{k}}\left(\left(\frac{s_{k}^{T} \bar{B}_{k} s_{k}}{s_{k}^{T} z_{k}}\right)^{1 / 2} z_{k}-\bar{B}_{k} s_{k}\right)^{T}, \\
& \bar{L}_{k}=J_{k+1}+\frac{\left\|r\left(x_{k+1}\right)\right\|^{2}}{\left\|r\left(x_{k}\right)\right\|} L_{k}, \\
& \bar{B}_{k}=\bar{L}_{k}^{T} \bar{L}_{k}, \\
& z_{k}=\left\|r\left(x_{k+1}\right)\right\|\left(J_{k+1}-J_{k}\right)^{T} \frac{r\left(x_{k+1}\right)}{\left\|r\left(x_{k}\right)\right\|}+J_{k+1}^{T} J_{k+1} s_{k} .
\end{aligned}
$$

The stepsize is computed by (4.1).

(6) Algorithm 2.1, denoted R-SFBFGS, where $B_{k}$ is updated by (4.3). Here we use the same parameters as R-FBFGS.

All codes were written in Matlab 7.4. In our experiments, we stopped whenever $\left\|g_{k}\right\|<10^{-4}$ or $f\left(x_{k}\right)-f\left(x_{k+1}\right) \leq 10^{-12} \max \left(1, f\left(x_{k+1}\right)\right)$ or the number of iterations exceeds $10^{4}$. In the methods R-FBFGS and R-SFBFGS, we set $L_{k+1}=0$ when $s_{k}^{T} z_{k}<10^{-20}$. The test problems are from [14]. Table-0 lists the name and related information of the test problems, where Z, S and L stand for zero residual, small residual and large residual problems, respectively. Tables 1-4 are numerical results of these methods, where

- iter(it1) is the number of iterations(the number of iterations using the line search (2.4) for the methods R-FBFGS and R-SFBFGS);

- $f n$ and normg are the number of the function evaluations and the norm of the gradient at the stopping point, respectively; 


\begin{tabular}{|c|c|c|c|c|c|}
\hline $\begin{array}{l}\text { Abbreviated } \\
\text { name }\end{array}$ & Name of test problem & $\mathrm{n}$ & $\mathrm{m}$ & starting point & Residual \\
\hline ROSE & Rosenbrock & 2 & 2 & $(-1.2,1)^{T}$ & $Z$ \\
\hline FROTH & Freudenstein and Roth & 2 & 2 & $(0.5,-2)^{T}$ & S \\
\hline BEALE & Beale & 2 & 3 & $(1,1)$ & Z \\
\hline JENSAM2 & Jennrich and Sampson & 2 & 2 & $(0.3,0.4)^{T}$ & $\mathrm{~S}$ \\
\hline JENSAM10 & Jennrich and Sampson & 2 & 10 & $(0.3,0.4)^{T}$ & $\mathrm{~L}$ \\
\hline KOWOSB & Kowalik and Osborne & 4 & 11 & $(0.25,0.39,0.415,0.39)^{T}$ & S \\
\hline $\mathrm{BD}$ & Brown and Dennis & 4 & 20 & $(25,5,-5,-1)^{T}$ & $\mathrm{~L}$ \\
\hline OSB2 & Osborne 2 & 11 & 65 & $(1.3,0.65, \cdots, 4.5,5.5)^{T}$ & S \\
\hline WATSON & Watson function & 20 & 31 & $(0,0, \cdots, 0)^{T}$ & S \\
\hline ROSEX & Extended Rosenbrock & 10 & 10 & $(-1.2,1, \cdots,-1.2,1)^{T}$ & $\mathrm{Z}$ \\
\hline SINGX & Extended Powell Singular & 20 & 20 & $(3,-1,0,1, \cdots, 3,-1,0,1)^{T}$ & S \\
\hline VARDIM & Variably Dimensioned & 10 & 12 & $(1-1 / n, \cdots, 1-n / n)^{T}$ & Z \\
\hline BAND & Broyden Banded & 10 & 10 & $(-1, \cdots,-1)^{T}$ & Z \\
\hline LIN1 & Linear Rank 1 & 10 & 10 & $(1, \cdots, 1)^{T}$ & S \\
\hline
\end{tabular}

Table 0 - Test problems.

- $f v$ and Av stand for the functional evaluation at the stopping point and the average of corresponding measure index, respectively;

- * means that the number of iterations exceeds $10^{4}$;

- Sp is the starting point for the problems BD, VARDIM and KOWOSB. Here we use seven different starting points, that is, $x 1=\left(10^{3}, \cdots, 10^{3}\right)^{T}$, $x 2=\left(10^{2}, \cdots, 10^{2}\right)^{T}, x 3=\left(10^{1}, \cdots, 10^{1}\right)^{T}, x 4=(1, \cdots, 1)^{T}, x 5=$ $\left(10^{-1}, \cdots, 10^{-1}\right)^{T}, x 6=\left(10^{-2}, \cdots, 10^{-2}\right)^{T}, x 7=\left(10^{-3}, \cdots, 10^{-3}\right)^{T}$.

As can be seen in Table 2, the method R-SFBFGS performed best for the problem $\mathrm{BD}$ since it requires the least number of iterations and function evaluations. The method R-FBFGS was faster than the methods LM and GN. Table 2 also shows that the method LM may not converge for this large residual problem since it fails to solve this problem within $10^{4}$ iterations when chosen the starting points $x 1$ and $x 2$. Tables 1 and 3 indicate that the method GN was the most efficient method for zero residual problems.

From Tables 1-4, we can see that the methods R-FBFGS and R-SFBFGS were stablest, which shows that both methods can ultimately converge to some local 


\begin{tabular}{|c|c|c|c|c|c|c|c|c|}
\hline \multirow[b]{2}{*}{ Problem } & \multicolumn{4}{|c|}{ R-FBFGS } & \multicolumn{4}{|c|}{ R-SFBFGS } \\
\hline & iter(it1) & $\mathrm{fn}$ & normg & $\mathrm{fv}$ & iter(it1) & fn & normg & $\mathrm{fv}$ \\
\hline ROSE & $27(0)$ & 41 & $1.7177 \mathrm{e}-007$ & $1.2109 \mathrm{e}-013$ & $26(0)$ & 42 & $1.4848 \mathrm{e}-006$ & $1.104 \mathrm{e}-011$ \\
\hline FROTH & $32(0)$ & 88 & 0.00043829 & 48.9843 & $31(0)$ & 74 & 0.00052402 & 48.9843 \\
\hline BEALE & $11(0)$ & 13 & $5.8368 \mathrm{e}-006$ & $2.2389 \mathrm{e}-010$ & $10(0)$ & 12 & $3.429 \mathrm{e}-007$ & $7.4291 \mathrm{e}-013$ \\
\hline JENSAM2 & $16(0)$ & 312 & 1.0965 & 0.27941 & $20(0)$ & 340 & 1.3805 & 0.28746 \\
\hline JENSAM 10 & $7(0)$ & 190 & 36522.7336 & 3437.4932 & $6(0)$ & 154 & 36523.336 & 3437.5617 \\
\hline KOWOSB & $11(0)$ & 13 & $3.3492 \mathrm{e}-005$ & 0.00030753 & $10(0)$ & 18 & $1.0464 \mathrm{e}-005$ & 0.00030751 \\
\hline $\mathrm{BD}$ & $404(3)$ & 2671 & 0.45658 & 85822.2016 & $417(3)$ & 2756 & 0.41606 & 85822.2016 \\
\hline OSB2 & $44(1)$ & 57 & $2.6039 \mathrm{e}-005$ & 0.040138 & $48(0)$ & 56 & $4.4437 \mathrm{e}-005$ & 0.040138 \\
\hline WATSON & $22(0)$ & 31 & $5.985 \mathrm{e}-006$ & $1.0197 \mathrm{e}-007$ & $19(0)$ & 20 & $3.1376 \mathrm{e}-006$ & $1.0195 \mathrm{e}-007$ \\
\hline ROSEX & $80(0)$ & 86 & $4.1689 \mathrm{e}-005$ & $2.4834 \mathrm{e}-009$ & $54(0)$ & 57 & $7.2284 \mathrm{e}-005$ & $1.8273 \mathrm{e}-008$ \\
\hline SINGX & $59(0)$ & 66 & $5.9403 \mathrm{e}-005$ & $9.5247 \mathrm{e}-007$ & $44(0)$ & 50 & $5.4738 \mathrm{e}-005$ & $8.5634 \mathrm{e}-007$ \\
\hline VARDIM & 19(7) & 20 & $7.5927 \mathrm{e}-008$ & $8.697 \mathrm{e}-016$ & $18(6)$ & 19 & $4.1247 \mathrm{e}-006$ & $3.5516 \mathrm{e}-012$ \\
\hline BAND & $28(0)$ & 29 & $6.8749 \mathrm{e}-005$ & $1.6857 \mathrm{e}-010$ & $24(0)$ & 25 & $9.575 \mathrm{e}-006$ & $1.7626 \mathrm{e}-012$ \\
\hline \multirow[t]{2}{*}{ LIN1 } & $1(1)$ & 2 & $1.3036 \mathrm{e}-010$ & 2.1429 & $1(1)$ & 2 & $1.3036 \mathrm{e}-010$ & 2.1429 \\
\hline & \multicolumn{4}{|c|}{ FBFGS } & \multicolumn{4}{|c|}{ SFBFGS } \\
\hline Problem & iter & fn & normg & $\mathrm{fv}$ & iter & fn & normg & $\mathrm{fv}$ \\
\hline ROSE & 14 & 45 & $4.8858 \mathrm{e}-014$ & $5.7192 \mathrm{e}-030$ & 14 & 44 & 0 & 0 \\
\hline FROTH & 6 & 156 & 53.5088 & 56.9355 & 11 & 212 & 53.047 & 56.7989 \\
\hline BEALE & 1 & 2 & $\mathrm{NaN}$ & $\mathrm{NaN}$ & 1 & 2 & $\mathrm{NaN}$ & $\mathrm{NaN}$ \\
\hline JENSAM2 & 24 & 474 & 1.9419 & 0.30837 & 29 & 609 & 2.2511 & 0.32263 \\
\hline JENSAM10 & 6 & 162 & 36522.7421 & 3437.4942 & 8 & 230 & 36523.3442 & 3437.5626 \\
\hline KOWOSB & 8 & 21 & $5.7009 \mathrm{e}-005$ & 0.00030752 & 9 & 22 & $2.0593 \mathrm{e}-006$ & 0.00030751 \\
\hline BD & 423 & 3219 & 0.38046 & 85822.2016 & 344 & 2609 & 0.44772 & 85822.2016 \\
\hline OSB2 & 17 & 40 & $1.6917 \mathrm{e}-005$ & 0.040138 & 20 & 44 & $2.1078 \mathrm{e}-005$ & 0.040138 \\
\hline WATSON & 15 & 41 & $3.6602 \mathrm{e}-005$ & $1.0194 \mathrm{e}-007$ & 9 & 22 & $3.6417 \mathrm{e}-006$ & $1.0196 \mathrm{e}-007$ \\
\hline ROSEX & 14 & 45 & $6.0032 \mathrm{e}-014$ & $1.6936 \mathrm{e}-029$ & 14 & 44 & $7.8129 \mathrm{e}-014$ & $1.2634 \mathrm{e}-029$ \\
\hline SINGX & 12 & 13 & $3.5266 \mathrm{e}-005$ & $9.4964 \mathrm{e}-008$ & 11 & 12 & $7.9839 \mathrm{e}-005$ & $6.5463 \mathrm{e}-007$ \\
\hline VARDIM & 16 & 17 & $4.5149 \mathrm{e}-007$ & $5.2809 \mathrm{e}-016$ & 15 & 16 & $1.569 \mathrm{e}-008$ & $6.3777 \mathrm{e}-019$ \\
\hline BAND & 58 & 585 & 0.14979 & 0.00072902 & 9 & 10 & $2.8327 \mathrm{e}-005$ & $1.7317 \mathrm{e}-011$ \\
\hline \multirow[t]{2}{*}{ LIN1 } & 1 & 2 & $\mathrm{NaN}$ & $\mathrm{NaN}$ & 1 & 2 & $\mathrm{NaN}$ & $\mathrm{NaN}$ \\
\hline & \multicolumn{4}{|c|}{ GN } & \multicolumn{4}{|c|}{ LM } \\
\hline Problem & iter & $\mathrm{fn}$ & normg & $\mathrm{fv}$ & iter & $\mathrm{fn}$ & normg & $\mathrm{fv}$ \\
\hline ROSE & 11 & 38 & $4.9452 \mathrm{e}-014$ & $4.9797 \mathrm{e}-030$ & 22 & 32 & $2.1751 \mathrm{e}-005$ & $1.8964 \mathrm{e}-009$ \\
\hline FROTH & 10 & 262 & 57.3339 & 58.1129 & 79 & 443 & 0.0013233 & 48.9843 \\
\hline BEALE & 1 & 2 & $\mathrm{NaN}$ & $\mathrm{NaN}$ & 9 & 10 & $7.7481 \mathrm{e}-006$ & $1.7642 \mathrm{e}-010$ \\
\hline JENSAM2 & 29 & 602 & 2.4368 & 0.33211 & 18 & 318 & 2.6138 & 0.34176 \\
\hline JENSAM10 & 4 & 129 & 36533.6555 & 3438.7345 & 10 & 203 & 32704.789 & 3007.2856 \\
\hline KOWOSB & 8 & 12 & $3.0796 \mathrm{e}-005$ & 0.00030756 & 8 & 9 & $2.7402 \mathrm{e}-005$ & 0.00030754 \\
\hline $\mathrm{BD}$ & 398 & 3020 & 0.57759 & 85822.2016 & 6573 & 62665 & 0.18377 & 85822.2016 \\
\hline OSB2 & 8 & 14 & $1.827 \mathrm{e}-005$ & 0.040138 & 41 & 45 & $9.7864 \mathrm{e}-005$ & 0.040138 \\
\hline WATSON & 4 & 5 & $1.3935 \mathrm{e}-007$ & $1.0194 \mathrm{e}-007$ & 20 & 21 & $2.1749 \mathrm{e}-006$ & $1.0194 \mathrm{e}-007$ \\
\hline ROSEX & 11 & 38 & $1.1058 \mathrm{e}-013$ & $2.4898 \mathrm{e}-029$ & 54 & 59 & $1.0544 \mathrm{e}-005$ & $5.3372 \mathrm{e}-011$ \\
\hline SINGX & 8 & 9 & $3.0164 \mathrm{e}-005$ & $1.8743 \mathrm{e}-007$ & 78 & 79 & $2.9682 \mathrm{e}-005$ & $4.0319 \mathrm{e}-007$ \\
\hline VARDIM & 9 & 10 & $7.8389 \mathrm{e}-007$ & $1.5919 \mathrm{e}-015$ & 30 & 31 & $3.1339 \mathrm{e}-006$ & $2.1246 \mathrm{e}-012$ \\
\hline BAND & 8 & 9 & $3.0316 \mathrm{e}-005$ & $1.8754 \mathrm{e}-011$ & 21 & 22 & $5.9486 \mathrm{e}-005$ & $7.2724 \mathrm{e}-011$ \\
\hline LIN1 & 1 & 2 & $\mathrm{NaN}$ & $\mathrm{NaN}$ & 1 & 2 & $5.5977 \mathrm{e}-010$ & 2.1429 \\
\hline
\end{tabular}

Table 1 - Test results for test problems with given points in Table-0. 


\begin{tabular}{|c|c|c|c|c|c|c|c|c|}
\hline & \multicolumn{4}{|c|}{ R-FBFGS } & \multicolumn{4}{|c|}{ R-SFBFGS } \\
\hline $\mathrm{Sp}$ & iter(it1) & fn & normg & $f v$ & iter(it1) & fn & normg & $f v$ \\
\hline $\mathrm{x} 1$ & $408(11)$ & 2659 & 0.35844 & 85822.2016 & $263(11)$ & 1630 & 0.46197 & 85822.2016 \\
\hline $\mathrm{x} 2$ & $427(9)$ & 2794 & 0.49511 & 85822.2016 & $321(8)$ & 2091 & 0.52185 & 85822.2016 \\
\hline $\mathrm{x} 3$ & $372(2)$ & 2436 & 0.38249 & 85822.2016 & $453(3)$ & 2924 & 0.35598 & 85822.2016 \\
\hline $\mathrm{x} 4$ & 271(3) & 1728 & 0.50631 & 85822.2016 & $397(2)$ & 2619 & 0.34217 & 85822.2016 \\
\hline $\mathrm{x} 5$ & $357(3)$ & 2366 & 0.2513 & 85822.2016 & $380(4)$ & 2561 & 0.38891 & 85822.2016 \\
\hline$x 6$ & 299(3) & 1975 & 0.41898 & 85822.2016 & $298(2)$ & 1950 & 0.13986 & 85822.2016 \\
\hline $\mathrm{x} 7$ & $357(1)$ & 2362 & 0.33776 & 85822.2016 & $360(2)$ & 2348 & 0.27332 & 85822.2016 \\
\hline \multirow[t]{2}{*}{$\mathrm{Av}$} & $355.9(4.6)$ & 2331.4 & & & 353.1(4.6) & 2303.3 & & \\
\hline & \multicolumn{4}{|c|}{ FBFGS } & \multicolumn{4}{|c|}{ SFBFGS } \\
\hline $\mathrm{x} 1$ & 432 & 3235 & 0.24956 & 85822.2016 & 444 & 3312 & 0.28299 & 85822.2016 \\
\hline $\mathrm{x} 2$ & 376 & 2824 & 0.57481 & 85822.2016 & 384 & 2894 & 0.35015 & 85822.2016 \\
\hline $\mathrm{x} 3$ & 448 & 3392 & 0.42223 & 85822.2016 & 434 & 3268 & 0.36913 & 85822.2016 \\
\hline $\mathrm{x} 4$ & 335 & 2515 & 0.19002 & 85822.2016 & 319 & 2407 & 0.33001 & 85822.2016 \\
\hline $\mathrm{x} 5$ & 320 & 2448 & 0.41839 & 85822.2016 & 326 & 2461 & 0.07763 & 85822.2016 \\
\hline$x 6$ & 356 & 2710 & 0.26727 & 85822.2016 & 303 & 2330 & 0.36326 & 85822.2016 \\
\hline $\mathrm{x} 7$ & 332 & 2481 & 0.13457 & 85822.2016 & 344 & 2599 & 0.39983 & 85822.2016 \\
\hline \multirow[t]{2}{*}{ Av } & 371.3 & 2800.7 & & & 364.9 & 2753 & & \\
\hline & \multicolumn{4}{|c|}{ GN } & \multicolumn{4}{|c|}{ LM } \\
\hline $\mathrm{x} 1$ & 450 & 3388 & 0.33622 & 85822.2016 & $10000^{*}$ & 174105 & - & - \\
\hline $\mathrm{x} 2$ & 431 & 3246 & 0.32536 & 85822.2016 & $10000^{*}$ & 67699 & - & - \\
\hline $\mathrm{x} 3$ & 406 & 3034 & 0.32743 & 85822.2016 & 9694 & 86477 & 0.16024 & 85822.2016 \\
\hline $\mathrm{x} 4$ & 389 & 2943 & 0.40604 & 85822.2016 & 941 & 7944 & 0.15998 & 85822.2016 \\
\hline $\mathrm{x} 5$ & 437 & 3298 & 0.42836 & 85822.2016 & 286 & 2098 & 0.27745 & 85822.2016 \\
\hline$x 6$ & 381 & 2908 & 0.3431 & 85822.2016 & 393 & 2853 & 0.25431 & 85822.2016 \\
\hline $\mathrm{x} 7$ & 340 & 2504 & 0.44166 & 85822.2016 & 485 & 3547 & 0.18451 & 85822.2016 \\
\hline Av & 404.9 & 3045.9 & & & 4542.7 & 49246 & & \\
\hline
\end{tabular}

Table 2 - Test results for the large residual problem BD.

stationary points for the given test problems. Moreover, we observe that the line search (2.4) was rarely used, but it is very efficient for the large residual problems.

In order to show the number of iterations or function evaluations performance of the six methods more clearly, we made Figures 1-2 according to the dada in Tables 1-4 by using the performance profiles of Dolan and Moré [7].

Since the top curve in Figures 1-2 corresponds to GN when $\tau<1$, this method is clearly the most efficient method for this set of 35 test problems. GN needs the least number of iterations and function evaluations for about $48 \%$ and $51 \%$ of the test problems. The possible reason is that most test problems are zero or very small residual problems. However, GN only solves $87 \%$ of the test 


\begin{tabular}{|c|c|c|c|c|c|c|c|c|}
\hline \multirow[b]{2}{*}{ Sp } & \multicolumn{4}{|c|}{ R-FBFGS } & \multicolumn{4}{|c|}{ R-SFBFGS } \\
\hline & iter(it1) & $\mathrm{fn}$ & normg & $\mathrm{fv}$ & iter(it1) & fn & normg & $f v$ \\
\hline $\mathrm{x} 1$ & $29(19)$ & 30 & $7.3573 \mathrm{e}-006$ & $1.1704 \mathrm{e}-011$ & $34(20)$ & 43 & $6.4822 \mathrm{e}-007$ & $1.1197 \mathrm{e}-015$ \\
\hline $\mathrm{x} 2$ & $25(16)$ & 31 & $9.2223 \mathrm{e}-008$ & $2.9985 \mathrm{e}-017$ & $24(16)$ & 25 & $1.05 \mathrm{e}-005$ & $2.3794 \mathrm{e}-011$ \\
\hline $\mathrm{x} 3$ & $21(12)$ & 22 & $3.4701 \mathrm{e}-006$ & $5.381 \mathrm{e}-013$ & $21(12)$ & 22 & $5.2768 \mathrm{e}-005$ & $5.8634 \mathrm{e}-010$ \\
\hline $\mathrm{x} 4$ & $0(0)$ & 1 & 0 & 0 & $0(0)$ & 1 & 0 & 0 \\
\hline $\mathrm{x} 5$ & $19(7)$ & 20 & $9.4672 \mathrm{e}-007$ & $1.7041 \mathrm{e}-013$ & $18(7)$ & 19 & $6.9588 \mathrm{e}-005$ & $7.4793 \mathrm{e}-010$ \\
\hline $\mathrm{x} 6$ & $20(8)$ & 21 & $1.7441 \mathrm{e}-006$ & $6.403 \mathrm{e}-014$ & 19(7) & 20 & $5.7122 \mathrm{e}-005$ & $4.2315 \mathrm{e}-010$ \\
\hline $\mathrm{x} 7$ & $20(8)$ & 21 & $6.0293 \mathrm{e}-006$ & $4.2892 \mathrm{e}-013$ & $20(7)$ & 21 & $2.4841 \mathrm{e}-008$ & $1.3298 \mathrm{e}-016$ \\
\hline \multirow[t]{2}{*}{$\mathrm{Av}$} & 19.1(10) & 20.9 & & & $19.4(9.9)$ & 21.6 & & \\
\hline & \multicolumn{4}{|c|}{ FBFGS } & \multicolumn{4}{|c|}{ SFBFGS } \\
\hline $\mathrm{x} 1$ & 29 & 30 & $5.1336 \mathrm{e}-005$ & $6.8275 \mathrm{e}-012$ & 30 & 31 & $4.8478 \mathrm{e}-005$ & $6.0885 \mathrm{e}-012$ \\
\hline $\mathrm{x} 2$ & 26 & 27 & $1.5511 \mathrm{e}-005$ & $6.2332 \mathrm{e}-013$ & 26 & 27 & $4.2816 \mathrm{e}-005$ & $4.7493 \mathrm{e}-012$ \\
\hline $\mathrm{x} 3$ & 22 & 23 & $2.3744 \mathrm{e}-007$ & $1.4606 \mathrm{e}-016$ & 21 & 22 & $5.1305 \mathrm{e}-005$ & $6.8191 \mathrm{e}-012$ \\
\hline $\mathrm{x} 4$ & 0 & 1 & 0 & 0 & 0 & 1 & 0 & 0 \\
\hline $\mathrm{x} 5$ & 17 & 18 & $2.5966 \mathrm{e}-009$ & $1.7467 \mathrm{e}-020$ & 16 & 17 & $1.663 \mathrm{e}-011$ & $7.1643 \mathrm{e}-025$ \\
\hline $\mathrm{x} 6$ & 17 & 18 & $1.1484 \mathrm{e}-007$ & $3.4167 \mathrm{e}-017$ & 16 & 17 & $1.2009 \mathrm{e}-008$ & $3.7362 \mathrm{e}-019$ \\
\hline $\mathrm{x} 7$ & 10 & 11 & $5.8861 \mathrm{e}-012$ & $8.9756 \mathrm{e}-026$ & 45 & 46 & $9.7049 \mathrm{e}-007$ & $2.0374 \mathrm{e}-013$ \\
\hline \multirow[t]{2}{*}{ Av } & 17.3 & 18.3 & & & 22 & 23 & & \\
\hline & \multicolumn{4}{|c|}{ GN } & \multicolumn{4}{|c|}{ LM } \\
\hline $\mathrm{x} 1$ & 20 & 21 & $1.9919 \mathrm{e}-012$ & $1.0279 \mathrm{e}-026$ & $10000^{*}$ & 10001 & - & - \\
\hline $\mathrm{x} 2$ & 16 & 17 & $8.999 \mathrm{e}-006$ & $2.098 \mathrm{e}-013$ & 3152 & 3153 & $1.6329 \mathrm{e}-007$ & $5.7681 \mathrm{e}-015$ \\
\hline $\mathrm{x} 3$ & 13 & 14 & 7.7751e-010 & $1.5661 \mathrm{e}-021$ & 302 & 303 & $5.3062 \mathrm{e}-008$ & $6.0906 \mathrm{e}-016$ \\
\hline $\mathrm{x} 4$ & 0 & 1 & 0 & 0 & 0 & 1 & 0 & 0 \\
\hline $\mathrm{x} 5$ & 10 & 11 & 0 & 0 & 42 & 43 & $4.3908 \mathrm{e}-008$ & $4.1704 \mathrm{e}-016$ \\
\hline $\mathrm{x} 6$ & 10 & 11 & $3.8833 \mathrm{e}-012$ & $3.9067 \mathrm{e}-026$ & 45 & 46 & $4.7351 \mathrm{e}-008$ & $4.8502 \mathrm{e}-016$ \\
\hline $\mathrm{x} 7$ & 10 & 11 & $5.8861 \mathrm{e}-012$ & $8.9756 \mathrm{e}-026$ & 45 & 46 & $9.7049 \mathrm{e}-007$ & $2.0374 \mathrm{e}-013$ \\
\hline $\mathrm{Av}$ & 11.3 & 12.3 & & & 1940.9 & 1941.9 & & \\
\hline
\end{tabular}

Table 3 - Test results for the zero residual problem VARDIM.

problems successfully while the methods R-FBFGS and R-SFBFG can solve all test problems. For $\tau>2$, the top curves correspond to R-FBFGS and R-SFBFGS, which shows that both methods are best within a factor $\tau$ with respective to the number of iterations and function evaluations.

\section{Conclusions}

We have proposed a regularized factorized quasi-Newton method with a new Armijo-type line search for nonlinear least squares problems. Under suitable conditions, global convergence of the proposed method is established. We also compared the performance of the proposed method to some existing methods. Numerical results show that the proposed method is promising. 


\begin{tabular}{|c|c|c|c|c|c|c|c|c|}
\hline & \multicolumn{4}{|c|}{ R-FBFGS } & \multicolumn{4}{|c|}{ R-SFBFGS } \\
\hline $\mathrm{Sp}$ & iter(it1) & fn & normg & $f v$ & iter(it1) & fn & normg & $f v$ \\
\hline $\mathrm{x} 1$ & 12(3) & 73 & $2.2596 \mathrm{e}-005$ & 0.00094437 & $17(0)$ & 118 & $7.682 \mathrm{e}-007$ & 0.0010271 \\
\hline $\mathrm{x} 2$ & $23(0)$ & 145 & $2.408 \mathrm{e}-007$ & 0.00094199 & $134(0)$ & 1373 & $4.2586 \mathrm{e}-005$ & 0.0017947 \\
\hline $\mathrm{x} 3$ & $12(0)$ & 41 & $3.5145 \mathrm{e}-006$ & 0.00091307 & $14(0)$ & 85 & $9.1386 \mathrm{e}-005$ & 0.00091308 \\
\hline $\mathrm{x} 4$ & $11(0)$ & 29 & $5.3353 \mathrm{e}-005$ & 0.00063121 & $6(0)$ & 7 & $5.4055 \mathrm{e}-005$ & 0.00064601 \\
\hline $\mathrm{x} 5$ & $6(0)$ & 7 & $2.4862 \mathrm{e}-005$ & 0.00030753 & $6(0)$ & 7 & $1.6505 \mathrm{e}-005$ & 0.00030752 \\
\hline $\mathrm{x} 6$ & $18(0)$ & 47 & $6.5095 \mathrm{e}-005$ & 0.00030793 & $17(0)$ & 35 & $5.5261 \mathrm{e}-005$ & 0.00030754 \\
\hline $\mathrm{x} 7$ & $13(0)$ & 29 & $1.5021 \mathrm{e}-005$ & 0.00030758 & $18(0)$ & 56 & $7.3324 \mathrm{e}-005$ & 0.0003083 \\
\hline \multirow[t]{2}{*}{$\mathrm{Av}$} & $13.6(0.4)$ & 53 & & & $30.3(0)$ & 240.1 & & \\
\hline & \multicolumn{4}{|c|}{ FBFGS } & \multicolumn{4}{|c|}{ SFBFGS } \\
\hline $\mathrm{x} 1$ & 7 & 274 & 0.00015227 & 0.0074384 & 2 & 142 & 3.9349 & 9.9516 \\
\hline $\mathrm{x} 2$ & 2 & 110 & 68.0119 & 3084.3729 & 2 & 110 & 68.0119 & 3084.3729 \\
\hline $\mathrm{x} 3$ & 611 & 12675 & $9.969 \mathrm{e}-005$ & 0.0079896 & 510 & 9705 & $8.9072 \mathrm{e}-006$ & 0.00042368 \\
\hline $\mathrm{x} 4$ & 13 & 38 & $4.8556 \mathrm{e}-005$ & 0.00030753 & 10 & 22 & $6.4239 \mathrm{e}-005$ & 0.00030752 \\
\hline $\mathrm{x} 5$ & 6 & 7 & $2.1055 \mathrm{e}-006$ & 0.00030751 & 6 & 7 & $3.3314 \mathrm{e}-006$ & 0.00030751 \\
\hline $\mathrm{x} 6$ & 60 & 572 & $5.6015 \mathrm{e}-006$ & 0.00030751 & 10 & 25 & $9.7842 \mathrm{e}-005$ & 0.0016273 \\
\hline $\mathrm{x} 7$ & $10000^{*}$ & 241941 & 93.015 & 0.016707 & 7245 & 178138 & $9.9965 \mathrm{e}-005$ & 0.0026686 \\
\hline \multirow[t]{2}{*}{$\mathrm{Av}$} & 1528.4 & 36517 & & & 1112.1 & 26878 & & \\
\hline & \multicolumn{4}{|c|}{ GN } & \multicolumn{4}{|c|}{ LM } \\
\hline $\mathrm{x} 1$ & 8 & 48 & $3.5482 \mathrm{e}-005$ & 0.0077006 & 108 & 1042 & $4.8297 \mathrm{e}-007$ & 0.0010271 \\
\hline $\mathrm{x} 2$ & 9 & 71 & $9.9007 \mathrm{e}-005$ & 0.14258 & 1445 & 36235 & 0.53282 & 0.13116 \\
\hline $\mathrm{x} 3$ & 53 & 635 & $7.6567 \mathrm{e}-005$ & 0.0010352 & 70 & 793 & $1.5315 \mathrm{e}-006$ & 0.0017946 \\
\hline $\mathrm{x} 4$ & 9 & 11 & $6.3432 \mathrm{e}-005$ & 0.00030769 & 6 & 8 & $5.6632 \mathrm{e}-005$ & 0.0006283 \\
\hline $\mathrm{x} 5$ & 6 & 7 & $3.6309 \mathrm{e}-005$ & 0.00030758 & 6 & 7 & $8.2719 \mathrm{e}-006$ & 0.00030751 \\
\hline $\mathrm{x} 6$ & 6 & 10 & $8.366 \mathrm{e}-005$ & 0.0003076 & 10 & 17 & $6.8217 \mathrm{e}-006$ & 0.00030752 \\
\hline $\mathrm{x} 7$ & $10000^{*}$ & 240170 & 85.8983 & 0.015786 & 11 & 25 & $6.2113 \mathrm{e}-006$ & 0.00030752 \\
\hline $\mathrm{Av}$ & 1441.6 & 34422 & & & 236.6 & 5446.7 & & \\
\hline
\end{tabular}

Table 4 - Test results for the small residual problem KOWOSB.

Acknowledgement. Part work of the first author was done when he was visiting Hirosaki University. This work was partially supported by the NSF foundation (10901026) of China, a project (09B001) of Scientific Research Fund of Hunan Provincial Education Department, the Hong Kong Polytechnic University Postdoctoral Fellowship Scheme and a scholarship from the Japanese Government.

\section{REFERENCES}

[1] M. Al-Baali and R. Fletcher, Variational methods for non-linear least squares. J. Oper. Res. Soc., 36 (1985), 405-421.

[2] R.H. Byrd and J. Nocedal, A tool for the analysis of quasi-Newton methods with application to unconstrained minimization. SIAM J. Numer. Anal., 26 (1989), 727-739.

[3] R.H. Byrd, J. Nocedal and Y. Yuan, Global covergence of a class of quasi-Newton method on convex problens. SIAM J. Numer. Anal., 24 (1987), 1171-1190. 


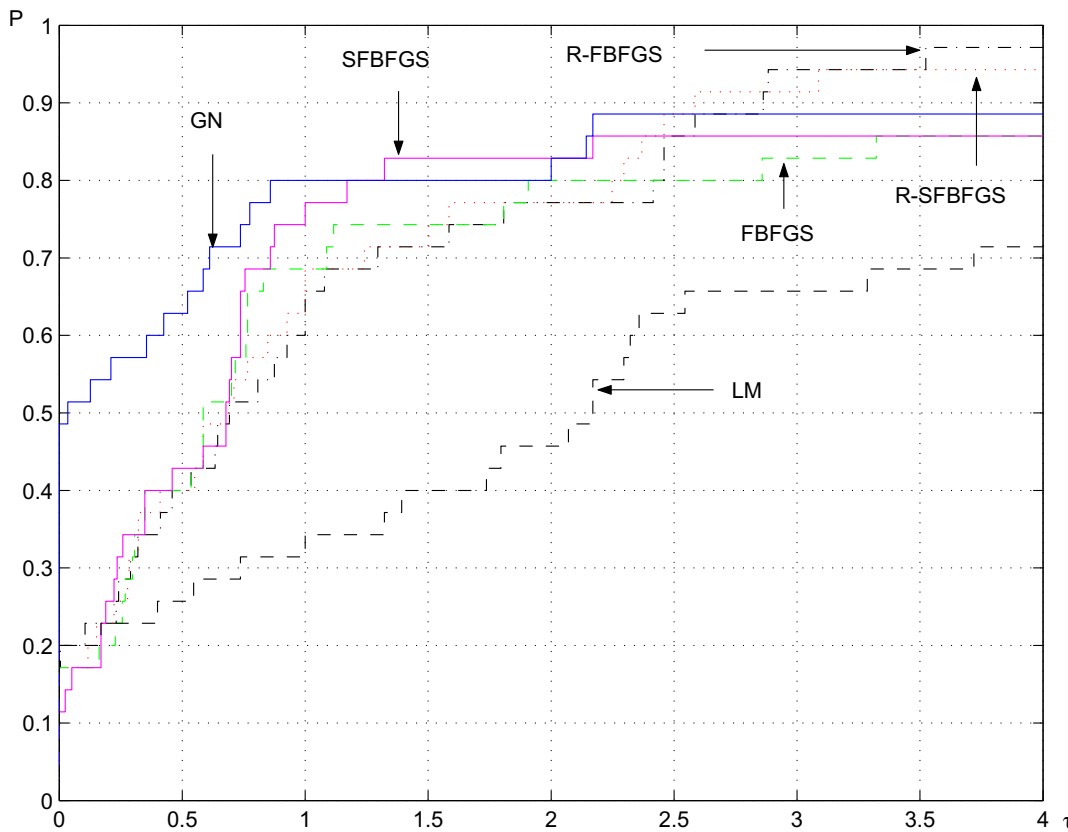

Figure 1 - Performance profiles with respect to the number of iterations.

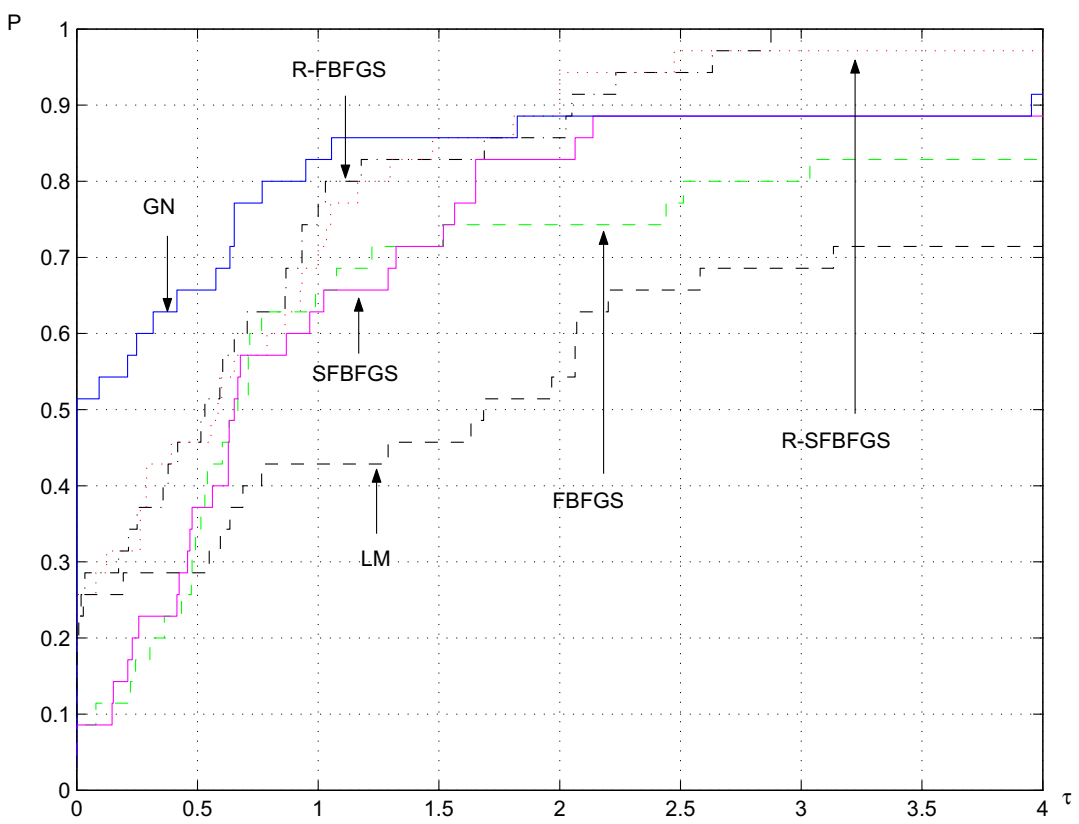

Figure 2 - Performance profiles with respect to the number of function evaluations. 
[4] Y.H. Dai, Convergence properties of the BFGS algorithm. SIAM J. Optim., 13 (2002), 693-701.

[5] J.E. Dennis, H.J. Martínez and R.A. Tapia, Convergence theory for the structured BFGS secant method with application to nonlinear least squares. J. Optim. Theory Appl., 61 (1989), $161-178$.

[6] J.E. Dennis and H.J. Schnabel, Numerical methods for unconstrained optimization and nonlinear equations. Prentice-Hall, Englewood Cliffs, NJ (1983).

[7] E.D. Dolan and J.J. Moré, Benchmarking optimization software with performance profiles. Math. Program., 91 (2002), 201-213.

[8] J.R. Engels and H.J. Martínez, Local and superlinear for partially known quasi-Newton methods. SIAM J. Optim., 1 (1991), 42-56.

[9] J. Fan and Y. Yuan, On the quadratic convergence of the Levenberg-Marquardt method without nonsingularity assumption. Computing, 74 (2005), 23-39.

[10] J. Huschens, On the use of product structure in secant methods for nonlinear least squares problems. SIAM J. Optim., 4 (1994), 108-129.

[11] D.H. Li and M. Fukushima, A modified BFGS method and its global convergence in nonconvex minimization. J. Comput. Appl. Math., 129 (2001), 15-35.

[12] T.W. Liu, BFGS method and its application to constrained optimization. Ph.D. thesis, College of Mathematics and Econometrics, Hunan University, Changsha, China (2007).

[13] W.F. Mascarenhas, The BFGS method with exact line searches fails for non-convex objective functions. Math. Program., 99 (2004), 49-61.

[14] J.J. Moré, B.S. Garbow, K.E. Hillstrom, Testing unconstrained optimization software. ACM Trans. Math. Softw., 7 (1981), 17-41.

[15] H. Ogasawara, A note on the equivalence of a class of factorized Broyden families for nonlinear least squares problems. Linear Algebra Appl., 297 (1999), 183-191.

[16] H. Ogasawara and H. Yabe, Superlinear convergence of the factorized structured quasiNewton methods for nonlinear optimization. Asia-Pacific J. Oper. Res., 17 (2000), 55-80.

[17] M.J.D. Powell, On the convergence of the variable metric algorithms. J. Inst. Math. Appl., 7 (1971), 21-36.

[18] C.X. Xu, X.F. Ma and M.Y. Kong, A class of factorized quasi-Newton methods for nonlinear least squares problems. J. Comput. Math., 14 (1996), 143-158.

[19] H. Yabe and T. Takahashi, Structured quasi-Newton methods for nonlinear least squares problems. TRU Math., 24 (1988), 195-209.

[20] H. Yabe and T. Takahashi, Factorized quasi-Newton methods for nonlinear least squares problems. Math. Program., 51 (1991), 75-100. 
[21] H. Yabe and N. Yamaki, Convergence of a factorized Broyden-like family for nonlinear least squares problems. SIAM J. Optim., 5 (1995), 770-791.

[22] H. Yabe and N. Yamaki, Local and superlinear convergence of structured quasi-Newton methods for nonlinear optimization. J. Oper. Res. Soc. Japan, 39 (1996), 541-557.

[23] N. Yamashita and M. Fukushima, On the rate of convergence of the Levenberg-Marquardt method. Computing (Supp.), 15 (2001), 237-249.

[24] J.Z. Zhang, L.H. Chen and N.Y. Deng, A family of scaled factorized Broyden-like methods for nonlinear least squares problems. SIAM J. Optim., 10 (2000), 1163-1179.

[25] W. Zhou and L. Zhang, Global convergence of the nonmonotone MBFGS method for nonconvex unconstrained minimization. J. Comput. Appl. Math., 223 (2009), 40-47.

[26] W. Zhou and D. Li, A globally convergent BFGS method for nonlinear monotone equations without any merit functions. Math. Comput., 77 (2008), 2231-2240. 